\title{
Review of: "Forensic Identification of Fentanyl and its Analogs by Electrochemical-Surface Enhanced Raman Spectroscopy (EC-SERS) for the Screening of Seized Drugs of Abuse"
}

\author{
Jasenka Gajdoš Kljusurić
}

Potential competing interests: The author(s) declared that no potential competing interests exist.

In a series of information and methods for drug detection, it is interesting to find a scientifically based approach to forensic identification of fentanyl and its analogues by Raman spectroscopy enhanced electrochemical surface spectroscopy (ECSERS) to verify seized drug abuse. The research focused on the method developed and presented in this paper that enables in-situ preparation of SERS substrates by providing a fast, efficient and accurate approach to fentanyl detection, even at low weight concentrations common in seized drugs.

What should certainly be emphasized is the willingness of the author of the paper to highlight potential limitations (which should certainly be borne in mind in in-situ methods and SARS, NIR, FTIR and similar methods). Qualitatively extremely helpful, and quantitatively it will certainly be confirmed through a series of future research. 